To the Editors:

\title{
Leiomyosarcoma of the inferior vena cava
}

\section{Introduction}

A 34-year old woman with an 8-month history of nonspecific abdominal pain was diagnosed as having a leiomyosarcoma of the inferior vena cava (IVC). Complete resection of the tumour with reconstruction of the IVC was done, followed by postoperative chemo-radiotherapy. Less than 200 cases of leiomyosarcoma of IVC are reported in publications up to date. This is the first reported case of complete resection of an IVC tumour in Sri Lanka.

\section{Case report}

The patient was admitted in August 2004 with a history of generalised, episodic, gradually worsening abdominal pain and backache. A large mass was palpable extending from the right costal margin to right iliac fossa. No distended veins were visible on the abdomen. The mass was firm, irregular, non-tender, mobile and nonpulsatile. The physical examination was otherwise unremarkable and rectal examination was normal.

Contrast and plain CT scan of the abdomen showed a large right-sided retroperitoneal mass involving the IVC at the level of the renal vein. It seemed well demarcated and was separate from the right kidney. The IVC was dilated up to $4.1 \mathrm{~cm}$ at the level of the tumour suggesting presence of tumour within the IVC. The retro-hepatic IVC was ill-defined suggesting probable involvement. There was no evidence of tumour extension into the lower IVC. An intravenous urogram was normal.

An exploratory laparotomy was done via a Chevron incision extended in the midline up to the xiphisternum. A large $(15 \times 16 \times 9 \mathrm{~cm})$ mass was identified retroperitoneally arising from the IVC at the level of right renal vein. Tumour was involving the IVC along a length of $5 \mathrm{~cm}$ upwards from the right renal vein and the lumen was filled with tumour. No tumour deposits were found elsewhere in the peritoneal cavity.

The tumour was excised with the part of the IVC wall that was involved. Reconstruction of the IVC was achieved with $4 / 0$ polypropylene.

Post-operative recovery was unremarkable and the patient was referred for chemo-radiotherapy. Histology revealed fascicles of spindle cells with eosinophilic cytoplasm and elongated cigar-shaped nuclei. Mitotic figures were numerous. The resection margins were free of tumour. The histological features were compatible with grade II leiomyosarcoma of IVC.
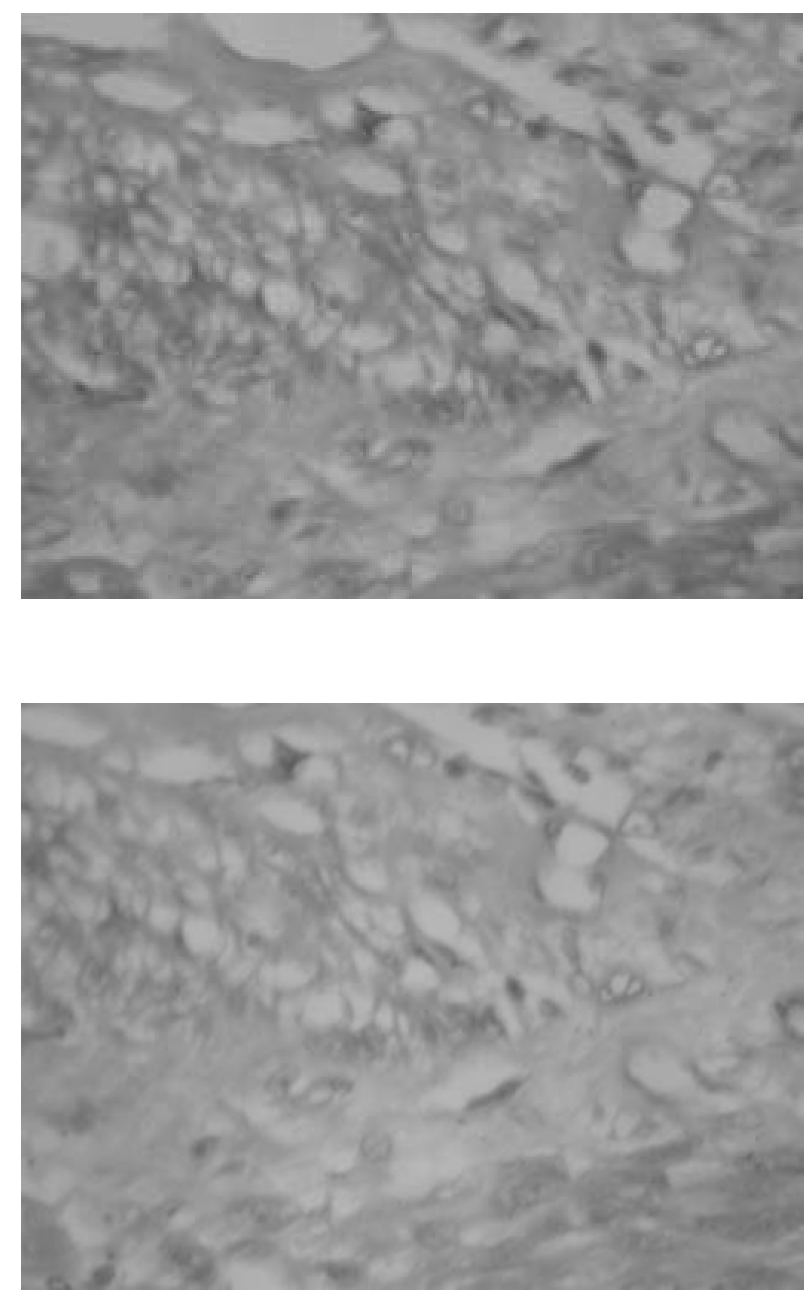

\section{Discussion}

Leiomyosarcoma of the IVC is a rare malignant tumour originating from the smooth muscle of the media. This tumour shows a striking preponderance for older women, with a male to female ratio of $1: 5$. The differential diagnosis of an intraluminal mass in the IVC includes leiomyosarcoma, angiosarcoma, tumour thrombus and bland thrombus. Complete resection is often possible in the lower segment. In the middle segment a more complicated resection along with the right kidney is usually needed if the renal vein is involved. Complete resection is usually not possible in upper segment due to extension into the hepatic veins and right side of the heart. The reported resectability of an IVC tumour is $40 \%$ to $60 \%$, but the prognosis is poor. The local recurrence rate is about $36 \%$, most patients die within 3 years, and the 5year survival rate is about $30 \%$. 
Symptoms and resectability depend on the location and extension of the tumour as well as associated thrombosis. In general, patients with leiomyosarcoma of the inferior vena cava have non-specific complaints such as malaise, weight loss, and abdominal and back pain.

\section{References}

1. Van Rooij WJJ, Martens F, Verbeeten B, Dijkstra J. CT and MR imaging of leiomyosarcoma of inferior vena cava. Radiology 1982; 145: 99-103.
2. Ahluwalia A, Saggar K, Sandhu P, Gupta K. Primary leiomyosarcoma of inferior vena cava. Indian Journal of Radiology Imaging 2002; 12: 515-6.

3. Monig SP, Gawenda M, Erasmi H, Zieren J, Pichimaier H. Diagnosis, treatment and prognosis of leiomyosarcoma of the inferior vena cava. European Journal of Surgery 1995; 161: $231-5$.

4. Rasaratnam R, Gunatunge CK, Seneviratne RH. Leiomyosarcoma of the inferior vena cava. Ceylon Medical Journal 1998; 43: 41-2.

\section{K S D S Benaragama, C A H Liyanage, S D Rajamanthri and K Alagaratnam}

Department of General Surgery, National Hospital of Sri Lanka, Colombo.

Correspondence: KSDS, e-mail <kapila_shanka@yahoo.com>. Conflicts of interests: none declared. Received 21 November and revised version accepted 3 December 2007. 